\section{Internações por condições sensíveis à atenção primária: estudo de validação do SIH/SUS em hospital do Distrito Federal, Brasil, 2012}

\author{
Hospitalizations for ambulatory care-sensitive \\ conditions: validation study at a Hospital \\ Information System (SIH) in the Federal \\ District, Brazil, in 2012
}

Hospitalizaciones por condiciones sensibles en la atención primaria: estudio de validación del Sistema de Información Hospitalaria (SIH), en un hospital del Distrito Federal, Brasil, 2012

\begin{abstract}
Resumo
Descrevem-se as internações por condições sensíveis à atenção primária com foco nas doenças infecciosas e parasitárias (DIP) e valida-se o Sistema de Informações Hospitalares do Sistema Único de Saúde (SIH/SUS), para o registro das internações por condições sensíveis à atenção primária, em hospital do Distrito Federal, Brasil, em 2012. Estimaram-se sensibilidade (S), especificidade (E), valor preditivo positivo (VPP) e valor preditivo negativo (VPN) do SIH/SUS para registro de internações por condições sensíveis à atenção primária, tendo o prontuário como padrão-ouro. Houve 1.604 (19,6\%; IC95\%: 18,7-20,5) internações por condições sensíveis à atenção primária, e as principais DIP foram: infecção do rim e trato urinário; infecção da pele e tecido subcutâneo; gastroenterites infecciosas. As DIP foram causa principal de internações na faixa etária de 20 a 29 anos, tendo causado 28 óbitos. Estimou-se $S=70,1 \%$ (IC95\%: 60,5-79,7), E = 88,4\% (IC95\%: 85,6-91,2), VPP = 51,7\% (IC95\%: 42,7-60,7) e VPN=94,3\% (IC95\%: 92,2-96,4). Os achados de internações por condições sensíveis à atenção primária no hospital em questão foram similares aos de outros estudos, com destaque para as internações por DIP. O SIH/SUS foi mais específico do que sensível.
\end{abstract}

Atenção Primária à Saúde; Hospitalização; Avaliação em Saúde;

Estudos de Validação
Danyelle Monteiro Cavalcante 1

Maria Regina Fernandes de Oliveira 1,2

Tânia Cristina Morais Santa Bárbara Rehem 1 


\section{Introdução}

No contexto internacional, os indicadores da atividade hospitalar são estudados como marcadores de resultados da qualidade da atenção primária e de acesso ao cuidado ambulatorial 1,2. O indicador denominado Ambulatory Care Sensitive Conditions 2 representa um conjunto de problemas de saúde para os quais a efetiva ação da atenção primária em saúde diminuiria o risco de internações hospitalares ${ }^{3,4}$. No Brasil, este indicador foi adotado com a denominação internações por condições sensíveis à atenção primária, por meio de portaria ministerial, e é composto por 19 grupos de causas e diagnósticos de acordo com a Classificação Internacional de Doenças, 10a revisão (CID-10) 1. Analisando a lista brasileira, há vários diagnósticos do grupo de doenças infecciosas e parasitárias (DIP), as quais ainda constituem um problema de saúde pública no Brasil 5. Para que a lista de internações por condições sensíveis à atenção primária, portanto, possa fornecer subsídios para a implantação ou redirecionamento das políticas e ações, faz-se necessário que essas internações estejam expressas de forma fidedigna no Sistema de Informações Hospitalares do Sistema Único de Saúde (SIH/SUS) ${ }^{6}$. Assim, o presente estudo tem como objetivo descrever as internações por condições sensíveis à atenção primária com ênfase em DIP e validar o SIH/SUS, quanto ao registro das internações por essas condições, no Hospital Regional do Paranoá, Distrito Federal, Brasil, no ano de 2012.

\section{Métodos}

Estudo epidemiológico descritivo e estudo de validação de critério. Pesquisaram-se os registros de internações do período de 1 o de janeiro a 31 de dezembro de 2012, último ano com dados fechados, no SIH/SUS, quando do começo da análise. Foram considerados casos de internações por condições sensíveis à atenção primária os diagnósticos contemplados na lista brasileira.

Foram analisadas as internações, descrevendo-se o perfil por DIP, dentre as internações por condições sensíveis à atenção primária, segundo as variáveis: idade, sexo, local de residência, grupos de diagnóstico, tempo de internação e tipo de alta. Excluíram-se as internações por partos, assumindo que não são doenças.

Para captar as internações por condições sensíveis à atenção primária no SIH/SUS, gerou-se arquivo de definição para tabulação, selecionando as causas de internações por meio dos respectivos códigos, no Tabwin, versão 3.6
(Departamento de Informática do SUS. http:// portal.saude.gov.br/portal/se/datasus/area. cfm?id_area=732). Os dados foram transferidos para o Excel (Microsoft Corp., Estados Unidos) para processamento, e a análise foi realizada no IBM SPSS, versão 20 (IBM Corp., Armonk, Estados Unidos). Foram calculadas frequências absolutas e relativas; a normalidade da distribuição foi testada por meio do teste Kolmogorov-Smirnov no nível de decisão a 5\%; foram calculadas as medianas para a as variáveis quantitativas.

Foram estimadas a sensibilidade, a especificidade, o valor preditivo positivo (VPP) e o valor preditivo negativo (VPN) do SIH/SUS para o registro adequado de internações por condições sensíveis à atenção primária, com seus respectivos intervalos de 95\% de confiança (IC95\%), com base nos diagnósticos de internações por condições sensíveis à atenção primária e demais causas. O padrão-ouro foi o prontuário.

Para a validação, foi realizada amostragem aleatória simples, considerando-se uma proporção com população finita, para a seleção dos prontuários; os parâmetros para os valores de sensibilidade e especificidade foram, respectivamente, 0,75 e 0,93 , baseados nos valores inferiores dos IC95\% do estudo de Rehem et al. 6 e com precisão de 0,04. Para a estimativa da sensibilidade do SIH, a amostra foi de 426 prontuários. Assumindo-se $20 \%$ de perdas, o número final foi 512 prontuários. Para a estimativa da especificidade, a amostra foi de 100 prontuários, e, considerando-se $20 \%$ de perda, 184 prontuários. Os 696 prontuários foram sorteados mediante lista de números aleatórios, tendo, como código para o sorteio, o número da Autorização de Internação Hospitalar (AIH). A extração dos diagnósticos dos prontuários eletrônicos foi realizada pela pesquisadora principal, com o auxílio direto de técnicos da gerência de informações do hospital.

Por meio do SPSS 20, foi gerada a lista de números aleatórios e, pelo Excel, foi feito o cálculo amostral. Para o processamento e análise dos dados, foram percorridas as etapas desenvolvidas no estudo de validação de Rehem et al. 6 .

O estudo foi aprovado pelo Comitê de Ética em Pesquisa da Fundação de Ensino e Pesquisa em Ciências da Saúde, com parecer 270.508, de 13 de maio 2013.

\section{Resultados}

No ano de 2012, excluindo-se partos, ocorreram $8.171(81,8 \%)$ internações gerais no Hospital Regional do Paranoá. Destas, 1.604 foram internações por condições sensíveis à atenção primária (19,6\%; IC95\%: 18,7-20,5), a maioria procedente 
do Distrito Federal: 1.554 (96,9\%), com mediana de permanência de três dias (intervalo: $<1-294$ dias).

Entre as internações por condições sensíveis à atenção primária, 724 (45,1\%) foram por DIP, com mediana de permanência de três dias (intervalo: < 1-144 dias). A mediana das idades dos pacientes internados foi 20 anos (intervalo: $<$ 1-97 anos), predominando o sexo feminino (471 casos; $65,1 \%)$.

Analisando as internações por DIP segundo faixa etária, verifica-se maior frequência de 20-29 anos, seguida pela faixa de 10-19 anos, com 145 (20\%) e 140 (19,3\%) internações respectivamente (Tabela 1).

Os quatro principais grupos de causas de internações por DIP foram: infecção do rim e trato urinário - 304 (42\%); infecção de pele e tecido subcutâneo - 167 (23,1\%); gastroenterites infecciosas e complicações - 109 (15,1\%); doenças das vias aéreas inferiores - 60 (8,3\%). Essas causas foram responsáveis por $88 \%$ do total de DIP registradas (Tabela 2).

Na análise segundo sexo, o feminino apresentou a maior frequência de internações no grupo das infecções do rim e trato urinário, com 263 $(55,8 \%)$ casos; por sua vez, no sexo masculino, a principal causa foi o grupo infecção da pele e tecido subcutâneo, com 99 (39,1\%) internações. Quanto ao tipo de alta, houve 664 (91,7\%) pacientes que se mantiveram internados em virtude de mudança para outros setores ou procedimentos, situação em que a AIH original é encerrada. Em segundo lugar, apareceram altas por transferências, com 32 (4,4\%) casos; em terceiro, por óbito, com 28 (3,9\%) ocorrências. Dentre as principais causas de óbitos por DIP, estão infecção de pele e tecido subcutâneo $(32,1 \%)$, seguida por gastroenterites infecciosas e suas complicações (28,6\%); em terceiro lugar, infecção do rim e trato urinário, com seis $(21,4 \%)$.

Para a validação do $\mathrm{SIH}$, foram estudados 577 prontuários devido à perda amostral (prontuários não localizados). A pesquisa aos prontuário identificou 87 internações por condições sensíveis à atenção primária e 490 internações por condições não sensíveis à atenção primária; a sensibilidade do SIH-SUS foi 70,1\% (IC95\%: 60,5-79,7); a especificidade, 88,4\% (IC95\%: 85,691,2); o VPP, 51,7\% (IC95\%: 42,7-60,7); o VPN, 94,3\% (IC95\%: 92,2-96,4) (Tabela 3). O SIH/SUS não registrou $30 \%$ das verdadeiras internações por condições sensíveis à atenção primária.

\section{Discussão}

Nesta pesquisa, os resultados encontrados para as internações por condições sensíveis à atenção primária foram semelhantes aos de outros estudos realizados no Brasil, com variações de $13,6 \%$ a $25,4 \% 7,8,9,10,11$. O acesso e a qualidade do serviço prestado pela atenção primária à saúde são pontos centrais nos resultados da análise das internações por condições sensíveis à atenção primária. Por outro lado, questões socioeconômicas e a prioridade concedida à oferta de serviços hospitalares, mesmo de baixa

Tabela 1

Internações por doenças infecciosas e parasitárias dentre as internações por condições sensíveis à atenção primária, segundo faixa etária e sexo. Hospital Regional do Paranoá, Distrito Federal, Brasil, 2012.

\begin{tabular}{|c|c|c|c|c|c|c|}
\hline \multirow[t]{2}{*}{ Faixa etária (anos) } & \multicolumn{2}{|c|}{ Feminino } & \multicolumn{2}{|c|}{ Masculino } & \multirow[t]{2}{*}{ Total } & \multirow[t]{2}{*}{$\%$} \\
\hline & $\mathbf{n}$ & $\%$ & $\mathrm{n}$ & $\%$ & & \\
\hline$<1$ & 2 & 0,3 & 5 & 0,7 & 7 & 1,0 \\
\hline $1-4$ & 66 & 9,1 & 72 & 9,9 & 138 & 19,1 \\
\hline $5-9$ & 31 & 4,3 & 38 & 5,2 & 69 & 9,6 \\
\hline $10-19$ & 109 & 15,0 & 31 & 4,3 & 140 & 19,3 \\
\hline $20-29$ & 120 & 16,6 & 25 & 3,5 & 145 & 20,0 \\
\hline $30-39$ & 70 & 9,7 & 32 & 4,4 & 102 & 14,1 \\
\hline $40-49$ & 23 & 3,2 & 12 & 1,6 & 35 & 4,8 \\
\hline $50-59$ & 14 & 1,9 & 13 & 1,8 & 27 & 3,7 \\
\hline 60 e mais & 36 & 5,0 & 25 & 3,5 & 61 & 8,4 \\
\hline Total & 471 & 65,1 & 253 & 34,9 & 724 & 100,0 \\
\hline
\end{tabular}

Fonte: Sistema de Informações Hospitalares do Sistema Único de Saúde (SIH/SUS). 
Tabela 2

Internações por doenças infecciosas e parasitárias dentre as internações por condições sensíveis à atenção primária, segundo grupo de diagnósticos e sexo, Hospital Regional do Paranoá, Distrito Federal, Brasil, 2012.

\begin{tabular}{|c|c|c|c|c|c|c|}
\hline \multirow[t]{2}{*}{ Grupos de diagnósticos } & \multicolumn{2}{|c|}{ Feminino } & \multicolumn{2}{|c|}{ Masculino } & \multirow[t]{2}{*}{ Total } & \multirow[t]{2}{*}{$\%$} \\
\hline & $\mathbf{n}$ & $\%$ & n & $\%$ & & \\
\hline 1. Doenças preveníveis por imunização e condições sensíveis & 5 & 1,1 & 10 & 3,9 & 15 & 2,1 \\
\hline 2. Gastroenterites infecciosas e complicações & 57 & 12,1 & 52 & 20,6 & 109 & 15,1 \\
\hline 5. Infecções do ouvido, nariz e garganta & 15 & 3,2 & 12 & 4,7 & 27 & 3,7 \\
\hline 6. Pneumonias bacterianas & 3 & 0,6 & 5 & 2,0 & 8 & 1,1 \\
\hline 8. Doenças das vias aéreas inferiores & 26 & 5,5 & 34 & 13,4 & 60 & 8,3 \\
\hline 15. Infecção do rim e trato urinário & 263 & 55,8 & 41 & 16,2 & 304 & 42,0 \\
\hline 16. Infecção da pele e tecido subcutâneo & 68 & 14,4 & 99 & 39,1 & 167 & 23,1 \\
\hline 17. Doença inflamatória de órgãos pélvicos femininos & 33 & 7,1 & 0 & 0,0 & 33 & 4,6 \\
\hline 19. Doenças relacionadas ao pré-natal e parto & 1 & 0,2 & 0 & 0,0 & 1 & 0,1 \\
\hline Total de internações por condições sensíveis à atenção primária & 471 & 100,0 & 253 & 100,0 & 724 & 100,0 \\
\hline
\end{tabular}

Fonte: Sistema de Informações Hospitalares do Sistema Único de Saúde (SIH/SUS).

Tabela 3

Classificação dos diagnósticos quanto às internações por condições sensíveis à atenção primária e não internações na Autorização de Internação Hospitalar (AlH) e no prontuário. Hospital Regional do Paranoá, Distrito Federal, Brasil, 2012.

\begin{tabular}{|c|c|c|c|}
\hline \multirow[t]{2}{*}{ Resultado } & \multicolumn{2}{|c|}{ Prontuário } & \multirow[t]{2}{*}{ Tota } \\
\hline & $\begin{array}{l}\text { Internações por condições } \\
\text { sensíveis à atenção primária }\end{array}$ & $\begin{array}{l}\text { Internações por condições não } \\
\text { sensíveis à atenção primária }\end{array}$ & \\
\hline \multicolumn{4}{|l|}{$\mathrm{AlH}$} \\
\hline Internações por condições sensíveis à atenção primária & 61 & 57 & 118 \\
\hline Internações por condições não sensíveis à atenção primária & 26 & 433 & 459 \\
\hline Total & 87 & 490 & 577 * \\
\hline
\end{tabular}

* Número final em virtude de perdas.

Fonte: Sistema de Informações Hospitalares do Sistema Único de Saúde (SIH/SUS) e prontuários do Hospital Regional do Paranoá.

qualidade, também podem contribuir para os resultados observados.

Quase metade das internações por condições sensíveis à atenção primária foram por DIP $(45,14 \%)$, realçando a influência deste grupo de doenças no perfil da morbimortalidade. Outro estudo no Distrito Federal identificou que as principais causas de internações por condições sensíveis à atenção primária foram gastroenterites, insuficiência cardíaca e infecção do rim e trato urinário ${ }^{10}$. Apesar da redução considerável no número de mortes por DIP nas últimas seis décadas, elas continuam sendo um problema de saúde pública no Brasil, determinando um forte impacto social e principalmente, econômico 12,13 .
O SIH/SUS apresentou maior probabilidade de fazer registros acurados de diagnósticos internações por condições não sensíveis à atenção primária, em comparação ao prontuário, do que de captar esses diagnósticos quando presentes. $\mathrm{O}$ sistema foi mais específico do que sensível, concordando com pesquisa anterior, de Rehem et al. 6 dado que deve ser levado em consideração na avaliação da atenção primária à saúde, não devendo o SIH-SUS ser a única fonte de informações para internações por condições sensíveis à atenção primária.

Estudos utilizando dados secundários podem sofrer limitações. No que se refere ao SIH/ SUS, as informações são restritas às ocorrências no SUS, o que poderia subnotificar alguns even- 
tos. Ademais, a investigação restringiu-se a um ano apenas e, no estudo de validação, houve perdas em quase $20 \%$, o que pode ter ocasionado redução da precisão para o dado de sensibilidade. É importante frisar que, para conhecimento mais ampliado das internações por condições sensíveis à atenção primária no Distrito Federal,

\section{Colaboradores}

D. M. Cavalcante participou do desenho da pesquisa, análise dos dados, redação do manuscrito e sua aprovação final. M. R. F. Oliveira e T. C. M. S. B. Rehem participaram do desenho da pesquisa, análise dos dados, redação do manuscrito e sua aprovação final. Foram responsáveis também pela revisão do trabalho. faz-se necessário analisar os fatores associados, tais como a condição socioeconômica dos usuários, devendo-se considerar, ainda, a qualidade e a cobertura da atenção primária, na região e no Distrito Federal, bem como as pactuações acerca da regulação das internações.

\section{Agradecimentos}

À direção e técnicos do Hospital Regional do Paranoá pela anuência e apoio técnico à execução da pesquisa.

\section{Referências}

1. Alfradique ME, Bonolo PF, Dourado I, Lima-Costa MF, Macinko J, Mendonça CS, et al. Internações por condições sensíveis à atenção primária: a construção da lista brasileira como ferramenta para medir o desempenho do sistema de saúde (Projeto ICSAP Brasil). Cad Saúde Pública 2009; 25:1337-49.

2. Billings J, Teicholz N. Uninsured patients in District of Columbia hospitals. Health Aff (Millwood) 1990; 9:158-65.

3. Starfield B. Atenção primária: equilíbrio entre necessidades de saúde, serviços e tecnologia. Brasília: Organização das Nações Unidas para Educação, Ciência e Cultura; 2002.

4. Caminal HJ, Morales EM, Sánchez-Ruiz E, CubellsLarrosa MJ, Bustins-Poblet M. Hospitalizaciones prevenibles mediante una atención primaria oportuna y efectiva. Aten Prim 2003; 31:6-14.

5. Barreto JOM, Nery IS, Costa MSC. Estratégia Saúde da Família e internações hospitalares em menores de 5 anos no Piauí, Brasil. Cad Saúde Pública 2012; 28:515-26.

6. Rehem TCMSB, Oliveira MRF, Ciosak SI, Egry EY. Registro das internações por condições sensíveis à atenção primária: validação do sistema de informação hospitalar. Rev Latnoam Enferm 2013; 21:1159-64.

7. Ferreira JBB, Borges MJG, Santos LL, Forster AC. Internações por condições sensíveis à atenção primária à saúde em uma região de saúde paulista, 2008 a 2010. Epidemiol Serv Saúde 2014; 23:45-56.

8. Rehem TCMSB, Ciosak SI, Egry EY. Internações por condições sensíveis à Atenção Primária no Hospital Geral de uma Microrregião de Saúde do Município de São Paulo, Brasil. Texto Contexto Enferm 2012; 21:535-42. 
9. Rehem TCMSB, Oliveira MRF, Amaral TCL, Ciosak SI, Egry EY. Internações por condições sensíveis à atenção primária em uma metrópole brasileira. Rev Esc Enferm USP 2013; 47:884-90.

10. Junqueira RMP, Duarte EC. Internações hospitalares por causas sensíveis à atenção primária no Distrito Federal, 2008. Rev Saúde Pública 2012; 46:761-8.

11. Pazó RG, Frauches DO, Galvêas DP, Stwfenoni AV, Cavalcante ELB, Pereira-Silva FH, et al. Internações por condições sensíveis à atenção primária no Espírito Santo: estudo ecológico descritivo no período 2005-2009. Epidemiol Serv Saúde 2012; 21:275-82.

\begin{abstract}
This study analyzes hospitalizations due to ambulatory care-sensitive conditions with a focus on infectious and parasitic diseases (IPDs) and validates the Hospital Information System, Brazilian Unified National Health System (SIH/SUS) for recording hospitalizations due to ambulatory care-sensitive conditions in a hospital in the Federal District, Brazil, in 2012. The study estimates the sensitivity, specificity, positive predictive value (PPV), and negative predictive value (NPV) of the SIH for recording hospitalizations due to ambulatory care-sensitive conditions, with the patient's medical file as the gold standard. There were 1,604 hospitalizations for hospitalizations due to ambulatory care-sensitive conditions (19.6\%, 95\%CI: 18.7-20.5), and the leading IPDs were renal and urinary tract infection, infection of the skin and subcutaneous tissue, and infectious gastroenteritis. IPDs were the leading cause of hospitalization in the 20 to 29-year age bracket and caused 28 deaths. Sensitivity was 70.1\% (95\%CI: 60.5-79.7), specificity $88.4 \%$ (95\%CI: 85.6-91.2), PPV = 51.7\% (95\%CI: 42.7-60.7), and NPV $=94.3 \%$ (95\%CI: 92.2-96.4). The findings for admissions due to ACSCs in this hospital were similar to those of other studies, featuring admissions for IPDs. The SIH/SUS database was more specific than sensitive.
\end{abstract}

Primary Health Care; Hospitalization;

Health Evaluation; Validation Studies
12. Departamento de Vigilância em Doenças Transmissíveis, Secretaria de Vigilância em Saúde, Ministério da Saúde. Plano integrado de ações estratégicas de eliminação da hanseníase, filariose, esquistossomose e oncocercose como problema de saúde pública, tracoma como causa de cegueira e controle das geohelmintíases: plano de ação 20112015. Brasília: Ministério da Saúde; 2012. (Série C. Projetos, Programas e Relatórios).

13. Ministério da Saúde. Morbidade hospitalar do SUS por local de residência: notas técnicas. http://tab net.datasus.gov.br/cgi/sih/nrdescr.htm (acessado em 20/Fev/2014).

\section{Resumen}

Se describen los internaciones por condiciones sensibles a la atención primaria, centrándose en las enfermedades infecciosas y parasitarias (DIP), y se valida el Sistema de Información Hospitalaria del Sistema Único de Salud (SIH/SUS) para el registro de las internaciones por condiciones sensibles a la atención primaria, en un hospital del Distrito Federal, en 2012. Estimadas sensibilidad (S), especificidad (E), valor predictivo positivo (VPP) y valor predictivo negativo (VPN) del SIH/SUS para el registro de internaciones por condiciones sensibles a la atención primaria, teniendo el historial como padrón-oro. Hubo 1.604 (19,6\%; IC95\%: 18,7-20,5) internaciones por condiciones sensibles a la atención primaria y las principales DIP fueron: infección del riñón y tracto urinario, infección de la piel y tejido subcutáneo y gastroenteritis infecciosas. Las DIP fueron la causa principal de las hospitalizaciones en la franja de edad de 20 a 29 años y causaron 28 óbitos. Se estimó $S=70,1 \%$ (IC95\%: $60,5-79,7), E=88,4 \%$ (IC95\%: 85,6-91,2), VPP = 51,7\% (IC95\%: 42,7-60,7) y $V P N=94,3 \%$ (IC95\%: 92,2-96,4). Los hallazgos de internaciones por condiciones sensibles a la atención primaria en este hospital fueron similares a otros estudios, destacando los internamientos por DIP. El SIH/ SUS fue más específico que sensible.

Atención Primaria de Salud; Hospitalización; Evaluación en Salud; Estudios de Validación
Recebido em 13/Nov/2014

Versão final reapresentada em 17/Jul/2015 Aprovado em 27/Ago/2015 